\title{
Ampere's magnetic circuital law: a simple and rigorous two-step proof
}

\author{
K. C. Rajaraman \\ Department of Electrical and Communication Engineering, Institut Teknologi Brunei, Bandar Seri Begawan, \\ Brunei Darussalam \\ E-mail: rajraman@itb.edu.bn
}

\begin{abstract}
A new proof of Ampere's law from the Biot-Savart law is presented. In the first step, a physical interpretation of current as moving charges carrying their electric fields with them simplifies the derivation of the magnetic field of current in a straight infinitely long conductor. The m.m.f. of a finite electric circuit linking a magnetic path is synthesized from those of two infinitely long wires carrying equal currents in opposite directions, only one of them threading the path. This makes the second step rigorous, enabling a non-mathematical treatment of the magnetic effects of electric currents in free space.
\end{abstract}

Keywords Ampere's law; electromagnetics; postulates; proof

List of symbols

(SI units are implied throughout)

$A$ area enclosed by current loop or circuit

$B$ magnetic flux density

$D$ electric flux density

$d$ distance between poles of dipole

$E \quad$ electric field strength

$f$ force of flux-current interaction

$H$ magnetic field strength

I current

$l$ length of magnetic circuit; length of conductor;

length (perpendicular to the page) of rectangular current loop

$m$ moment of magnetic dipole or magnetic shell

$p$ magnetic pole strength

$Q \quad$ electronic charge on current element

$q$ linear density of electronic charge on current element or conductor

$r$ distance of field point from field source

$U$ magnetic potential, m.m.f.

$v$ charge velocity in current element or conductor, electric-field velocity past field point

$w \quad$ width (in the plane of the page) of rectangular current loop

$\mu_{0} \quad$ permeability of free space

$\Omega$ solid angle subtended at a point by current circuit

This paper is about the way the topic of the magnetic effects of electric currents in free space is presented, with particular reference to the way Ampere's mag-

International Journal of Electrical Engineering Education 38/3 
netic circuital law is derived or proved, in an introductory course on engineering electromagnetics for junior undergraduates.

Carter $^{1}$ and Hammond and Sykulski ${ }^{2}$ start with the dipole/current-loop equivalence ('equivalence' for short) as the basic postulate and, through the concept of (scalar) magnetic potential, derive Ampere's and the Biot-Savart laws. Oatley ${ }^{3}$ and Duffin ${ }^{4}$ start with the Biot-Savart law and derive the equivalence, thence the magnetic potential due to a current loop and, finally, Ampere's law. Christopoulos, ${ }^{5}$ Sibley $^{6}$ and Kraus $^{7}$ use the Biot-Savart law to find the m.m.f. around a straight infinitely long current-carrying conductor and generalize it to Ampere's law.

Parts of these treatments are, at least for learners at this level, a little too complex in terms of the mathematics used, concepts required and the logic employed. Examples are Carter's deduction of the Biot-Savart law from magnetic potential ('if certain advanced mathematical methods be permitted'), ${ }^{1}$ Hammond and Sykulski's derivation of the Biot-Savart law from Ampere's ${ } \mathrm{aw}^{2}$ and Oatley's derivation of the equivalence from the Biot-Savart law. ${ }^{3}$

Reviewed in the next section, Ampere's law proofs themselves are only marginally less complex. Subsequent sections then present a simplified treatment using an optimum choice of postulates and of sequence of presentation. The treatment covers the whole topic with a minimum number of postulates.

\section{Existing proofs of Ampere's law}

From the dipole/current-loop equivalence

The equivalence is

$$
m=\mu_{0} I A
$$

With this starting point, there are at least two methods of proving Ampere's law, and each makes use of an additional result borrowed from electrostatics, by analogy.

In the first proof, given by Carter, ${ }^{1}$ Oatley ${ }^{3}$ and Duffin, ${ }^{4}$ the additional result used is that the magnetic (scalar) potential of a point due to a magnetic dipole can be written down by analogy with the already-known expression for the electric potential due to an electric dipole, as

$$
U=\frac{m \cos \theta}{4 \pi \mu_{0} r^{2}}
$$

where $\theta$ is the angle between the axis of the dipole and the direction of $r$, the line joining the centre of the dipole to the field point.

Imposing the equivalence, eqn (1), and recognizing that $(A \cos \theta) / r^{2}=\Omega$, the solid angle subtended by the equivalent current loop at the point, the potential due to the loop becomes $U=I \Omega / 4 \pi$. As the point moves once round a closed magnetic path linking the circuit, the solid angle described by it is $4 \pi$, so that the change in potential, or the m.m.f. acting in the path, is $I$.

International Journal of Electrical Engineering Education 38/3 
In the second proof, given by Hammond and Sykulski, ${ }^{2}$ the additional result used is that the magnetic potential difference between the two sides of a 'magnetic shell', equivalent to the current circuit, can be written down by analogy with the electric potential difference across the plates of a charged capacitor, as

$$
U=\frac{m}{\mu_{0} A}
$$

This is then shown to be equal to the change in potential in going round a closed path linking the circuit. Imposing the dipole/current-loop equivalence, eqn (1), this change in potential, or the m.m.f. acting in the path, becomes $I$.

Both proofs require the concept of the change in magnetic potential as an observer moves from one point to another. The process involved is implicitly mathematical, being the integration of $H$ over the path. But a physical interpretation is highly desirable at this level of teaching. Duffin ${ }^{4}$ says that, 'if a physical picture of this is required, the best we can do is to interpret it as the work done per unit pole in taking it round the path'. Hammond and Sykulski ${ }^{2}$ similarly say that, 'for a path linked with the current ... work is done by a unit pole traversing a current loop'. Thus the proofs involve movement of unit poles, an impossible process. Besides, the overall logic used by each is rather contrived and tortuous.

From the Biot-Savart law

This law, illustrated in Fig. 1(a), is:

$$
\delta H=\frac{I \delta l}{4 \pi r^{2}} \sin \theta
$$

The proof starting with this is a two-step procedure. In the first step, the magnetic field strength $H$ due to a straight conductor of finite length carrying current $I$ is found at a point at a perpendicular distance $r$. It is then extended

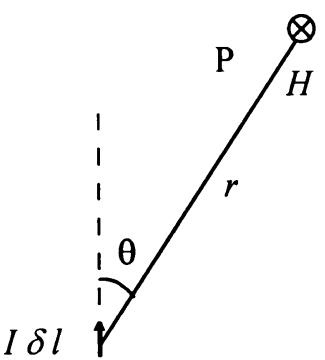

(a) Biot-Savart Law

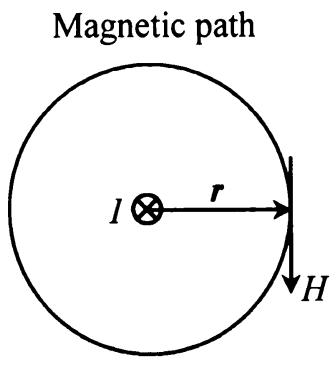

(b) $H$ around conductor

Fig. 1 Existing two-step proof.

International Journal of Electrical Engineering Education 38/3 
to a conductor of infinite length, obtaining

$$
H=\frac{I}{2 \pi r}
$$

The derivation is mathematical, involving a good deal of trigonometry and calculus. ${ }^{1,3-7}$ The m.m.f. acting in a closed circular path (say an iron ring, for ease of visualization), with the conductor at the centre, Fig. 1(b), is then obviously

$$
\oint H . d l=H . l=\frac{I}{2 \pi r} 2 \pi r=I
$$

or

$$
\oint H \cdot d l=I
$$

which is Ampere's law.

Note that this result has been obtained for the specific configuration of Fig. 1(b), i.e. for the following conditions:

(a) circular magnetic path; current through the centre of the path, and perpendicular to the plane of the path;

(b) straight and infinitely long conductor.

After arriving at this result, in the next step, the writers ${ }^{5-7}$ go on to generalize it. This they do by simply asserting that the result 'has been found to hold' not only for the geometry specified above, but also for all cases where the integration of $H$ is over a single closed magnetic path enclosing the conductor, i.e. irrespective of the actual configuration of the circuit or of the ring. This purported 'proof' is found not only in introductory texts (e.g. Christopoulos, ${ }^{5}$ Sibley $^{6}$ ) but also in more advanced treatises (e.g. Kraus ${ }^{7}$ ). As a statement of fact, this is of course true but, by 'the rules of the game', it needs to be rigorously proved.

Some authors (e.g. Christopoulos ${ }^{5}$ ) do address the question of condition (a) above, and show that, as long as the magnetic path encloses the conductor, neither the configuration of the path, nor the relative position of the conductor through it matters. (This is a fairly simple procedure, similar to showing that the work done by a charge in moving from one point to another in an electrostatic field is independent of the path taken.)

But what of condition (b): straight and infinitely long conductor? Fig. 2(a) shows a situation in which the circuit does not satisfy condition (b), i.e. is neither straight nor infinitely long, but obviously links with the ring (shown in sectional elevation with its plane horizontal). How can eqn (5), which was derived for a straight, infinitely long conductor, be applicable to Fig. 2(a)?

No writer seems to address this question. Duffin ${ }^{4}$ at least recognizes the problem, for he derives the equation, but apparently unable to generalize it to cover situations such as that of Fig. 2(a), rightly dismisses the result as a 'special 


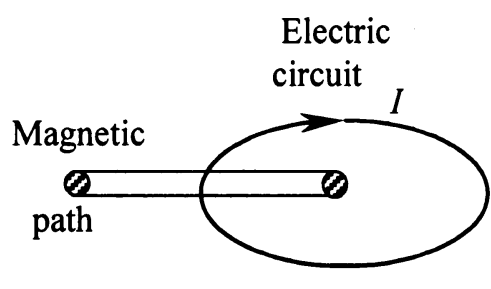

(a)

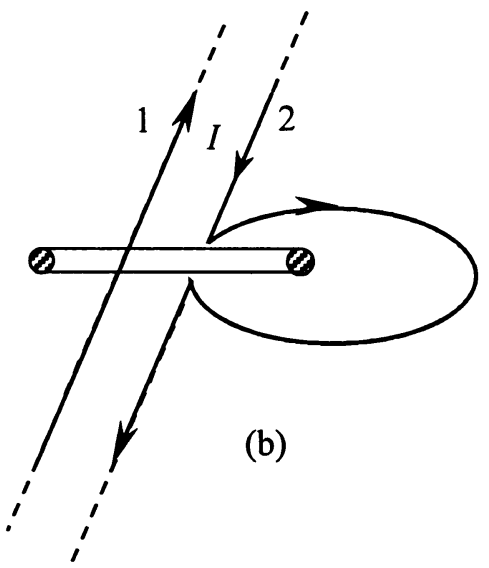

Fig. 2 Magnetic equivalence.

case'. He then goes on to give, ab initio, the first of the two more-general proofs that start with the dipole/current-loop equivalence as the basic postulate, outlined in the section 'From the dipole/current-loop equivalence' above.

The next two sections together present a unified sequence of derivations leading to Ampere's law. In the process, they constitute a simple, rigorous and complete treatment of the topic of magnetic effects of electric currents in free space.

\section{From the equivalence to the Biot-Savart law: a simplified treatment}

From the equivalence to the Lorentz force

Instead of eqn (2) or (3), let us borrow from electrostatics the expression for the force on a pole in a magnetic field

$$
f=p \cdot H
$$

by analogy with $f=q$. E, the force on a charge in an electric field.

Figure 3(a) shows a dipole and the equivalent (rigid rectangular) current loop, both situated in the same horizontal magnetic field with their axes parallel and at angle $\theta$ with the field.

Torque on the dipole $=p \cdot H d \sin \theta$

$$
=m H \sin \theta
$$

since $p . d=m$.

From the dipole/current-loop equivalence, eqn (1),

torque on the equivalent current loop $=\mu_{0} I A H \sin \theta$

$$
=B I l w \sin \theta
$$

International Journal of Electrical Engineering Education 38/3 


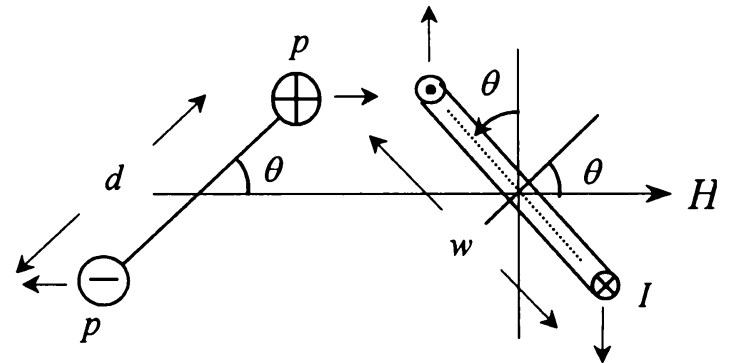

(a)

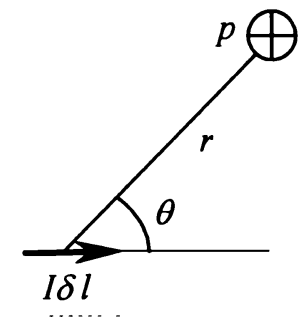

(b)

Fig. 3 From the equivalence to the Lorentz force.

since $\mu_{0} H=B$ and $A=l w$. Since $w \sin \theta$ is the separation between the two horizontal conductors of the loop, the force on each conductor acting in the directions shown must be

$$
f=B I l
$$

\section{From the Lorentz force to the Biot-Savart law ${ }^{6}$}

Figure 3(b) shows a magnetic pole and a current element, distance $r$ apart. If $\delta H$ is the strength of the magnetic field at the pole due to the current element, the force on the pole is, from eqn (6),

$$
\delta f=p \delta H
$$

acting out of the page.

By action and reaction, the current element, lying in the magnetic field of the pole, will experience the same force but in the opposite direction, i.e. into the page. The normal component of the flux density at the current element due to the pole is

$$
B=\frac{p}{4 \pi r^{2}} \sin \theta
$$

From equation (7), the force on the current element is

$$
\delta f=\frac{p}{4 \pi r^{2}} \sin \theta . I \delta l
$$

Equating the two expressions for $\delta f$,

$$
p \delta H=\frac{p}{4 \pi r^{2}} \sin \theta . I \delta l
$$

from which

$$
\delta H=\frac{I \delta l}{4 \pi r^{2}} \sin \theta
$$

which is the Biot-Savart law. 
[Note that the pole in equation (6) and that in Fig. 3(b) are stationary and their respective opposite poles are always present, distance $d$ away, but have merely been ignored. If necessary, their effect can easily be calculated and included in our study, but it has simply not been necessary. In any case, considering one pole of a stationary dipole is as legitimate as considering a current element. $\left.{ }^{2,6}\right]$

\section{The Biot-Savart law to Ampere's law: a new two-step proof}

Step 1: derivation of eqn (5) simplified

In Fig. 1(a), current being movement of the negative electrons in the negative direction,

$$
\begin{aligned}
I & =(-v)(-q) \\
& =v \cdot q
\end{aligned}
$$

(Alternatively, we may of course assume for simplicity that, notionally, current is constituted by movement of positive charges in the positive direction.)

The Biot-Savart law, eqn (4), then becomes

$$
\delta H=\frac{v q \delta l}{4 \pi r^{2}} \sin \theta
$$

But $q \delta l=\delta Q$, the total moving charge on the element.

Therefore

$$
\delta H=\frac{v \delta Q}{4 \pi r^{2}} \sin \theta
$$

But

$$
\frac{\delta Q}{4 \pi r^{2}}=\delta D
$$

the electric flux density at $\mathrm{P}$ due to $\delta Q$. Hence $\delta H=v . \delta D \sin \theta$.

Incorporating the relative directions using the vector notation, and generalizing,

$$
\boldsymbol{H}=\boldsymbol{v} \times \boldsymbol{D}
$$

Two points need to be settled at this stage. The first is that velocity $v$ is also that of the electric flux density $D$ past the field point P. (The usual controversy over a 'moving field' cannot arise here because the velocity is simply that of the charges to which the field is due.) The second point is that, in reality, there is no evidence of an electric field near a current-carrying conductor. This is because, while the moving electrons set up a stationary magnetic field, their travelling electric field is neutralized, at all points and at all times, by the stationary electric field of the positive charges at rest. ${ }^{8}$

International Journal of Electrical Engineering Education 38/3 
For the infinitely long conductor of Fig. 1 (b), obviously, $D=q / 2 \pi r$, radially outwards and moving axially into the page.

Hence

$H=\frac{v q}{2 \pi r}=\frac{I}{2 \pi r}$

Hence for the circular magnetic path with the conductor at the centre

$$
\oint H . d l=I
$$

\section{Step 2: rigorous generalization of eqn (5)}

In Fig. 2(b), circuit 1 is an infinitely long straight conductor passing through the ring in a direction tangential to the loop circuit of Fig. 2(a), carrying a current $I$ upwards. Circuit 2 carries the same value of current, but in the opposite direction; it also extends to infinity on either side of the ring, and runs parallel and infinitely close to circuit 1 (but shown apart for clarity) for the whole length, except at the ring itself.

Tracing the current path of circuit 2 from the top down, it can be seen that it approaches the ring, but just short of entering it, departs from the straightline path. Here it bends sharply backwards and traces a curved path identical to the loop circuit of Fig. 2(a). But again just short of entering the ring at the bottom, it returns to the straight-line path, rejoining conductor 1 and continuing the rest of the way.

Thus the straight-line part of circuit 2 differs from circuit 1 only in that the element that actually passes through the ring is missing. It is clear that circuit 2 , as a whole, does not link with the ring and hence will contribute no m.m.f. to it. Therefore, the m.m.f. in the ring due to both circuits 1 and 2 together is that due to circuit 1 alone, which is $I$.

Now consider the magnetic effects of the various sections of the two circuits. It can be seen that, over their entire straight lengths on both sides of the ring, the magnetic fields of the two circuits cancel each other. Magnetically, therefore, only the element of circuit 1 that actually passes through the ring plus the curved part of circuit 2 survive. But these two together obviously constitute the whole loop circuit of Fig. 2(a); in other words Figs 2(a) and 2(b) are magnetically equivalent. Therefore, the m.m.f. in the ring due to the loop circuit in Fig. 2(a) is equal to that of circuits 1 and 2 together in Fig. 2(b), which is simply $I$. This proves the generality of eqn (5), Ampere's law.

In effect, electromagnetically, the relation between a current circuit and a closed magnetic path is purely topological, i.e. the current either threads through the path or it does not, irrespective of the rest of their geometry. Where it does, the current contributes an m.m.f. equal to itself to the magnetic path.

International Journal of Electrical Engineering Education 38/3 


\section{Summary and general remarks}

All the postulates used and the laws derived in the paper are collected in Table 1. Column (3) contains eqn (7) and also an equivalent (in vector pointfunction form), eqn (9). Similarly, since Ampere's law has been directly derived from the Biot-Savart, without the need for any other postulate, the two laws become equivalent and, therefore, appear in the same column, column (4).

To summarize the procedure for deriving Ampere's law from the dipole/ current-loop equivalence, we start with the postulates in columns (1) and (2) and proceed across to column (3) and thence to the Biot-Savart law in column (4). Subsequently, and of course if starting with the Biot-Savart law itself, we simply proceed down the column, following the two-step procedure, to Ampere's law.

In the existing proofs starting with the equivalence, the 'thought experiment' of moving a unit magnetic pole around has to be visualized because a magnetic path has to be traced (through a given electric circuit). On the other hand, the proof presented here requires the tracing of only electric current paths (through and around a given magnetic circuit), a much more realistic process.

Compared to the existing two-step proof from the Biot-Savart law, a physical interpretation of current, in terms of moving charges carrying their electric fields with them, has made the first step, derivation of Ampere's law, eqn (5), much easier. As for the second step, the new proof has provided an elegant and non-mathematical, yet rigorous generalization of eqn (5), in the place of an unproven assertion.

Apart from these, the treatment presented has another advantage. A complete coverage, at this level, of the topic of the magnetic effects of electric currents in free space should include the Lorentz force, column (3) of Table 1: the force of flux-current interaction, eqn (7), and the e.m.f. of flux-cutting induction, eqn (9).

The existing treatments starting with the dipole/current-loop equivalence do not include the Lorentz force, and neither can it be derived from the two postulates used. Therefore, the force law will have to be separately postulated subsequently, making a total of three postulates to cover the whole topic.

TABLE 1 Postulates and laws

\begin{tabular}{|c|c|c|c|c|c|c|}
\hline \multicolumn{3}{|c|}{ Postulates } & \multicolumn{4}{|c|}{ Laws } \\
\hline $\begin{array}{l}\qquad(1) \\
\text { Dipole/current-loop } \\
\text { equivalence }\end{array}$ & $\begin{array}{l}\quad(2) \\
\text { Force on pole in } \\
\text { magnetic field }\end{array}$ & & Lorentz for & & $\begin{array}{l}\quad \text { (4) } \\
\text { Magnetic field of } \\
\text { electric current }\end{array}$ & \\
\hline$m=\mu_{0} I A$ & $f=p . H$ & (6) & $f=B I l$ & (7) & $\delta H=\frac{I \delta l}{4 \pi r^{2}} \sin \theta$ & (4) \\
\hline & & & $E=v \times B$ & (9) & $\begin{array}{l}\boldsymbol{H}=\boldsymbol{v} \times \boldsymbol{D} \\
\oint H . d l=I\end{array}$ & $\begin{array}{l}(8) \\
(5)\end{array}$ \\
\hline
\end{tabular}

International Journal of Electrical Engineering Education 38/3 
In comparison, the treatment presented here requires only two postulates, eqns (1) and (6), to cover all the laws: the Lorentz force, the Biot-Savart and Ampere's, in one unified sequence. [In fact, it can be shown that we only need equations from any two columns of Table 1, as long as at least one of them is a force formula from columns (2) or (3).]

\section{Conclusions}

Currently, even the simplest textbook treatments of the topic of the magnetic effects of electric currents in free space present one or more of the following problems to the learner: mathematical complexity, conceptual and logical difficulties, lack of rigour and too many basic postulates. In this paper, a judicious choice of postulates together with an optimum sequence of presentation have led to a simple, rigorous and complete treatment of the topic. This has been made possible by the new two-step proof of Ampere's magnetic circuital law.

\section{Acknowledgement}

The author learnt the essentials of electromagnetism from the late Prof. G. W. Carter (1909-1989) of Leeds University and from his classic book, The Electromagnetic Field in its Engineering Aspects. ${ }^{1}$

\section{References}

1 G. W. Carter, The Electromagnetic Field in its Engineering Aspects, 2nd edn (Longmans, 1967), pp. 84-85, 94-96.

2 P. Hammond and J. K. Sykulski, Electromagnetism: Physical Processes and Computation (Oxford University Press, Oxford, 1995), pp. 48-51, 58-60, 90-92.

3 C. W. Oatley, Electric and Magnetic Fields: An Introduction (Cambridge University Press, Cambridge, 1976), pp. 66-70, 74-75.

4 W. J. Duffin, Electricity and Magnetism, 4th edn (McGraw-Hill, London, 1990), pp. 195-196.

5 C. Christopoulos, An Introduction to Applied Electromagnetism (John Wiley \& Sons, Chichester, 1995), pp. 61-62, 65-66.

6 M. Sibley, Introduction to Electromagnetism (Arnold, 1996), pp. 69-70, 74-75.

7 J. D. Kraus, Electromagnetics, 4th edn (McGraw-Hill, New York, 1992), pp. 224-225, 238.

8 K. C. Rajaraman, 'Ampere and Faraday: the electromagnetic symmetry', Int. J. Elect. Enging. Educ., 33(4) (1996), 291-302. 